\title{
EVOLUTION AS A CONFOUNDING PARAMETER IN SCALING RELATIONS FOR GALAXIES
}

\author{
D. Fraix-Burnet ${ }^{1}$
}

\begin{abstract}
Early-type galaxies are characterized by many scaling relations. Evolutionary classifications find that some of these correlations are indeed generated by diversification. With a simple mathematical formalism, we show (Fraix-Burnet 2011) that even the so-called fundamental plane, a relatively tight correlation between three variables, can be easily explained as the artifact of the effect of another parameter influencing all, without any physical hypothesis. In other words, the fundamental plane is probably a confounding correlation, i.e. not physically causal. The complexity of the physics of galaxies and of their evolution suggests that the confounding parameter must be related to the level of diversification reached by the galaxies. Galaxy mass, central black hole mass or the gas fraction during the last big merger are shown to be possible confounding factors. Consequently, many scaling relations for galaxies are probably evolutionary correlations that are explained by the statistical general evolution of most properties of galaxies. This effect makes the observables not independent, so that it must be removed before statistical and physical inferences could be made.
\end{abstract}

Keywords: galaxies: fundamental parameters, methods: statistical, galaxies: evolution, galaxies: formation

\section{The fundamental plane as a confounding correlation}

The fundamental plane for early-type galaxies is a correlation between effective radius, the central velocity dispersion and the surface brightness within the effective radius (Diorgovski \& Davis 1987; Dressler et al. 1987). Let us consider that the effective radius $r_{e}$, the central velocity dispersion $\sigma$ and the luminosity $L$ are all powerlaw functions of a same generic parameter $\widetilde{X}$ :

$$
\left\{\begin{aligned}
r_{e} & =A_{1} \widetilde{X}^{p} \\
\sigma & =A_{2} \widetilde{X}^{s} \\
L & =A_{3} \widetilde{X}^{t}
\end{aligned}\right.
$$

The surface brightness $\mu_{e}$ can be expressed as

$$
\begin{aligned}
\mu_{e} & =-2.5 \log \left(L / \pi r_{e}^{2}\right)+m \\
& =(-2.5 t+5 p) \log \widetilde{X}+2.5 \log (\pi)+m
\end{aligned}
$$

where $m$ is a constant of normalisation. Any linear correlation of the form

$$
\log r_{e}=a \log \sigma+b \mu_{e}+c
$$

translates to

$$
\left\{\begin{aligned}
p & =s a+(-2.5 t+5 p) b \\
\log A_{1} & =a \log A_{2}+b\left(2.5 \log \left(\pi A_{1}^{2} / A_{3}\right)+m\right)+c .
\end{aligned}\right.
$$

If a solution can be found for $a$ and $b$ from Eq. 1.4 then the equation of the fundamental plane Eq. 1.3 is obtained. Conversely, the observations provide $a, b$ and $c$, so that it is possible to derive $p, s$ and $t$. There is no need of any further assumption to explain the fundamental plane.

\footnotetext{
${ }^{1}$ Université Joseph Fourier - Grenoble 1 / CNRS, Institut de Planétologie et d'Astrophysique de Grenoble, BP 53, F-38041

Grenoble cedex 9, France, e-mail: fraix@obs.ujf-grenoble.fr
} 
Here are a few examples for $\widetilde{X}$, the relations being constrained only by observations or numerical simulations and Eq. 1.4.

$$
\begin{aligned}
& \left\{\begin{array}{rll}
r_{e} & \propto & f_{\text {starburst }}^{-1} \\
\sigma & \propto & f_{\text {starburst }}^{1} \\
L & \propto & f_{\text {starburst }}^{0.8}
\end{array}\right. \\
& \left\{\begin{array}{rll}
r_{e} & \propto & M_{B H}^{0.63} \\
\sigma & \propto & M_{B H}^{0.28} \\
L & \propto & M_{B H}^{0.83}
\end{array}\right.
\end{aligned}
$$

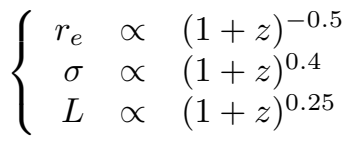

\section{Evolutionary correlations}

In the course of diversification, many properties of galaxies change, and they tend to statistically change in a more or less monotonous way. It seems difficult to avoid the evolution to act as a confounding factor. It is a well-known problem of comparative methods in phylogeny (e.g. Felsenstein 1985).
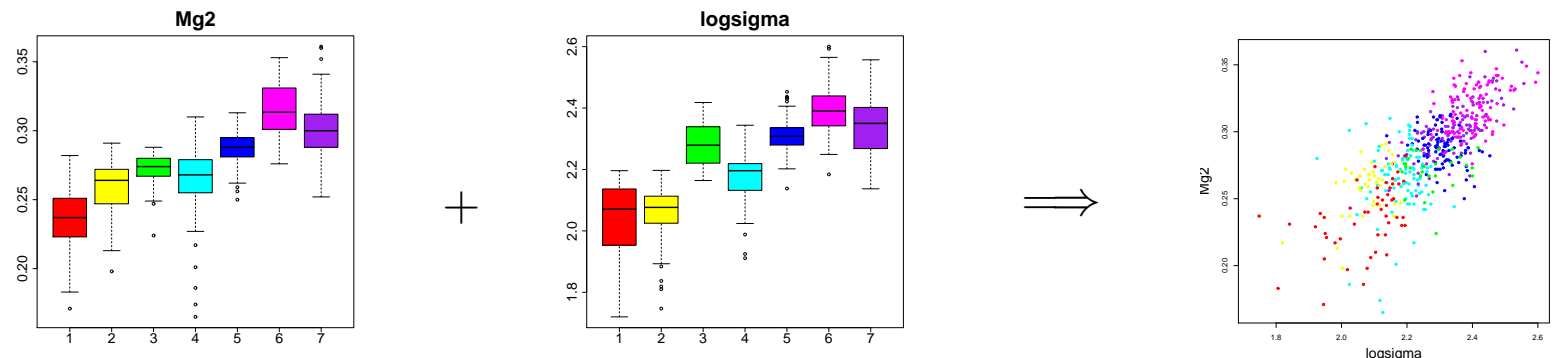

Fig. 1. Two parameters $\left(M g_{2}\right.$, left, and $\sigma$, middle) evolving as a function of an other parameter (here the level of diversification) necessarily produce a correlation in the corresponding scatter plot (right).

We thus propose that the main confounding parameter is

$$
\widetilde{X}=T
$$

with $T$ an indicator of the level of diversification, being something like an evolutionary clock not necessarily easily related to time or redshift.

Indeed, the evolutionary clock, i.e. the factor $\widetilde{X}=T$, can be hidden, not understandable analytically and not directly observable.

Diversification cannot be summarized with only one simple property (like redshift or mass) because galaxies are too complex objects and do not evolve linearly in a unique way. In some diagrams, that is for some set of variables, a particular property could crudely depict the general trend of diversification. In the case of $r_{e}$, $\sigma$ and $\mu_{e}$, and to a first approximation, mass could well represent a satisfactory driving parameter for the fundamental plane correlation, but it is certainly not unique. Since it is only approximate, some dispersion is expected. Hence, dispersion may be explained by the statistical (non-causal) nature of the correlation and the heterogeneity of the samples as far as diversification is concerned (Fraix-Burnet et al. 2010).

A lot of observables evolve with diversification, at least statistically, so that we should not be surprised by the many scaling relations found for galaxies and the difficulty to pinpoint the driving parameters and mechanisms. We also better understand why several characteristic parameters (mass, luminosity, metallicity...) and also the samples themselves have been found to influence the shape of the fundamental plane without providing a clearer picture of its origin. Physics can only be investigated when confounding factors have been eliminated. This here requires an evolutionary classification that gathers objects according to their history.

This work is published in Fraix-Burnet (2011). 


\section{Evolutionary correlation}

The evolution generates the correlation

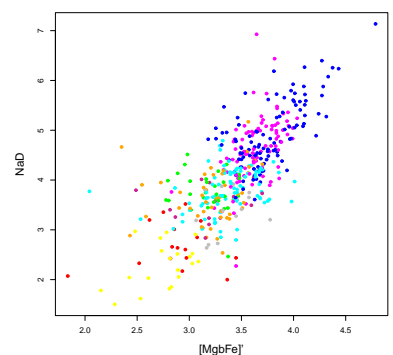

\section{Ordinary correlation}

The correlation may depend on the group

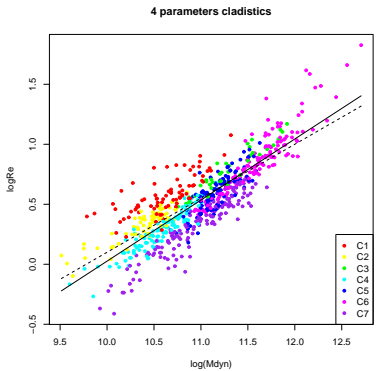

Fig. 2. Illustration of two kinds of correlations. On the left, the evolutionary correlation can be identified by the groups (indicated by their colors) nearly ordered according to their level of diversification. On the right, an ordinary correlation is independent of diversification, but may or may not exist globally and/or within each group.

\section{References}

Djorgovski, S. \& Davis, M. 1987, ApJ, 313, 59

Dressler, A., Lynden-Bell, D., Burstein, D., et al. 1987, ApJ, 313, 42

Felsenstein, J. 1985, The American Naturalist, 125, 1

Fraix-Burnet, D. 2011, MNRAS: Letters, 416, L36

Fraix-Burnet, D., Dugué, M., Chattopadhyay, T., Chattopadhyay, A. K., \& Davoust, E. 2010, MNRAS, 407, 2207 